\title{
Successful Treatment of Pemphigus Vulgaris With the Extensive Mucocutaneous Lesions in an Elderly Patient
}

\author{
Mohsen Masjedi ${ }^{1, *}$; Ali Asilian ${ }^{2}$; Zabihollah Shahmoradi ${ }^{2}$; Parvin Rajabi Dehnavi ${ }^{2}$; Bahareh \\ Abtahi Naeini ${ }^{2,3}$ \\ ${ }_{1}^{1}$ Department of Immunology, Faculty of Medicine, Isfahan University of Medical Sciences, Isfahan, IR Iran \\ ${ }^{2}$ Department of Dermatology, Faculty of Medicine, Isfahan University of Medical Sciences, Isfahan, IR Iran \\ ${ }^{3}$ Medicine Students' Research Committee, School of Medicine, Isfahan University of Medical Sciences, Isfahan, IR Iran \\ ${ }^{*}$ Corresponding Author: Mohsen Masjedi, Department of Immunology, Faculty of Medicine, Isfahan University of Medical Sciences, Isfahan, IR Iran. Tel: +98-9132705806, Fax: +98- \\ 3117922431, E-mail: masjedi@med.mui.ac.ir
}

Received: August 3, 2013; Revised: October 31, 2013; Accepted: January 28, 2014

\begin{abstract}
Introduction: Pemphigus vulgaris (PV) is a chronic and infrequent autoimmune mucocutaneous disease that is characterized by the loose blisters and erosions on the skin and mucous membrane. Middle-aged adults are affect most frequently and the elderly and juvenile cases are infrequent. Herein, we reported a case of pemphigus vulgaris in an elderly patient.

Case Presentation: We reported a case of pemphigus vulgaris in a 79-year-old patient with the extensive mucocutaneous lesions. We also reviewed the literature in MEDLINE with keywords such as pemphigus vulgaris, elderly, mucocutaneous lesions, oral lesions, and treatment.

Discussion: We have to stress that the importance of this case report is its presentation in an elderly patient, as the frequent age of presentation in the Iranian patients is the middle age. On the other hand, PV occurs rarely, thus, the reporting of any rare case with some exceptions is important.
\end{abstract}

Keywords:Pemphigus; Aged; Therapeutics

\section{Introduction}

Pemphigus vulgaris (PV) is an autoimmune vesiculobullous skin disease in which the immune system produces autoantibody against the specific proteins of the desmosomal adhesion complex that lead to the intraepidermal blister formation (1). The pathogenesis of this disease is not fully understood yet (2). PV is an infrequent disease; its incidence changes from 0.5 to 3.2 cases per 100000 individuals. Basically, PV covers an extensive age range with the peak frequency between the third and sixth decades of life (3). Although PV most frequently affects middle-aged adults, we report the case of a 79-year-old elderly patient with the extensive PV lesions who remitted completely with a combination therapy of oral prednisolone and azathioprine. Thus, we have to stress the importance of this case report in the elderly, as the mean age of presentation in the Iranian patients is the middle age, and not the elderly (4). On the other hand, PV occurs rarely (5); thus, reporting any rare case with some exceptions is of great importance.

\section{Case Presentation}

A 79-year-old man from Fereydunshahr Town, Isfahan Province, Iran, was referred to the Dermatology Clinic of
Al-Zahra Hospital of Isfahan with the bullous lesions on his head. Two months before the development of these lesions, the patient had lesions on his trunk, face, and gingivae. Neither of the common sites in the oral cavity were affected except the gingivae. There was no evidence of tense blisters and itch. There was no history of urticarial lesions. He had no history of using any medication (Table 1). Intraoral examination showed erosions and desquamation with the erythematous areas on the gingivae, which were not healing. Crusted plaques were also seen on his scalp, face, and trunk (Figures 1 and 2). With the suspicion of $\mathrm{PV}$, the biopsy specimen was taken from pemphigus lesions. Histopathological study of the skin lesion showed a suprabasal bulla containing some acantholytic cells. The basal cells rendered a tombstone appearance. In the dermis, there was a mild, superficial perivascular, lymphocytic infiltrate with a few eosinophils. The direct immunofluorescence (DIF) study also showed the characteristics deposition of IgG in the lace-like pattern. Hence, based on the clinical, histopathological, and immunofluorescence examination findings, the patient was diagnosed with PV (Table 1). Subsequently, he received system

\footnotetext{
Implication for health policy/practice/research/medical education:
}

Pemphigus vulgaris (PV) is a potentially fatal disease, particularly in the elderly. We presented a 79-year-old patient who went in remission following fouryear successful corticosteroids therapy. It shows good management of the long-term corticosteroids therapy adverse effects in this patient. In addition, $\mathrm{PV}$ is an immune-mediated disease and the role of autoantibodies in its pathogenesis is supported. Thus, corticosteroids therapy in this patient in spite of adverse effects would be also indicated that one of the main reasons for patient's remission was the downregulation of the immune system in the elderly. Copyright (C) 2014, Iranian Red Crescent Medical Journal; Published by Kowsar Corp. This is an open-access article distributed under the terms of the Creative Commons Attribution License, which permits unrestricted use, distribution, and reproduction in any medium, provided the original work is properly cited. 
Masjedi Met al.

\begin{tabular}{|c|c|}
\hline Variables & Description \\
\hline Age, $y$ & 79 \\
\hline Sex & Male \\
\hline Patient's History & No drug history, no urticarial lesions \\
\hline Clinical Findings & Lesions on the scalp; ulcer and hemorrhagic crust on the trunk \\
\hline Mucosal Lesions & Oral lesions: erosions and desquamation with the erythematous areas on the gingiva (desquamative gingivitis) \\
\hline $\begin{array}{l}\text { Pathological } \\
\text { Findings }\end{array}$ & $\begin{array}{l}\text { A suprabasal bulla containing some acantholytic cells. The basal cells rendered a "tombstone" appearance. In } \\
\text { the dermis, a mild, superficial perivascular, lymphocytic infiltrate with a few eosinophils were seen. }\end{array}$ \\
\hline DIF and IIF & IgG, in the dermis; deposition of IgG in the lace-like pattern \\
\hline Diagnosis & Pemphigus vulgaris \\
\hline Treatment & $\begin{array}{c}\text { The initial low-dose } 70-\mathrm{mg} / \mathrm{d} \text { oral prednisolone, then tapered to } 50 \mathrm{mg} / \mathrm{d} \text {, subsequently a } 5 \mathrm{mg} \text { reduction of } \\
\text { prednisolone every two weeks led to complete remission of lesions, The patient remained under the treatment } \\
\text { of } 10 \mathrm{mg} / \mathrm{d} \text { and } 100 \mathrm{mg} / \mathrm{d} \text { of prednisolone and azathioprine, respectively. }\end{array}$ \\
\hline
\end{tabular}

a Abbreviations: DIF, direct immunofluorescence; and IIF, indirect immunofluorescence.

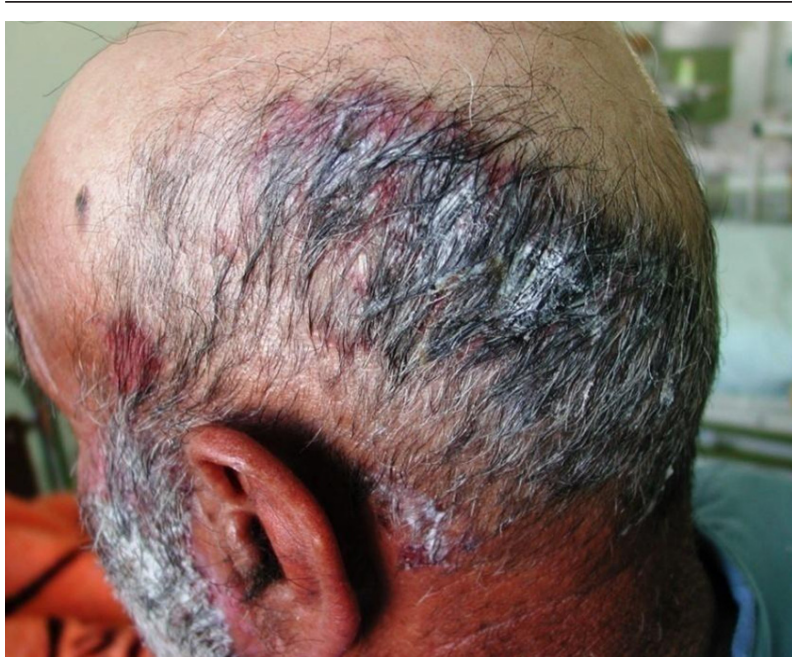

Figure 1. Pemphigus Vulgaris Lesions (Ulcer and Heavy Crust) on the Scalp

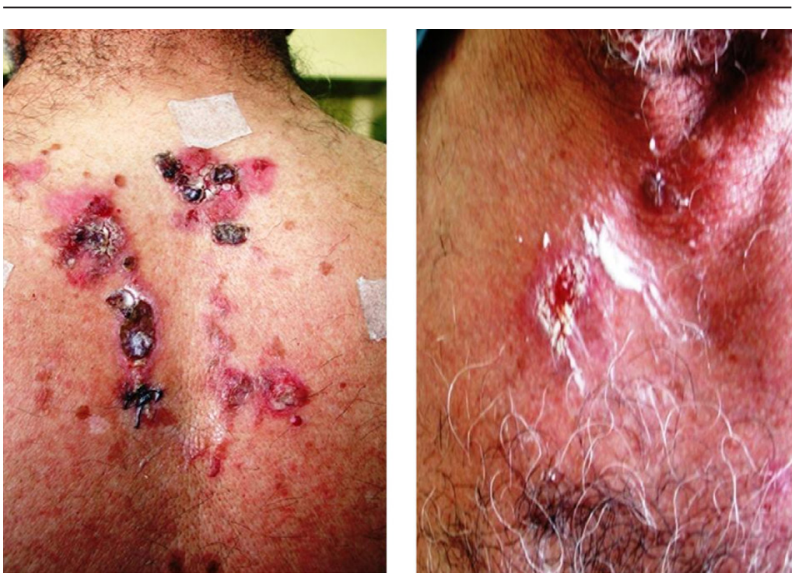

Figure 2. Pemphigus Vulgaris Ulcer and Hemorrhagic Crust on the Trunk

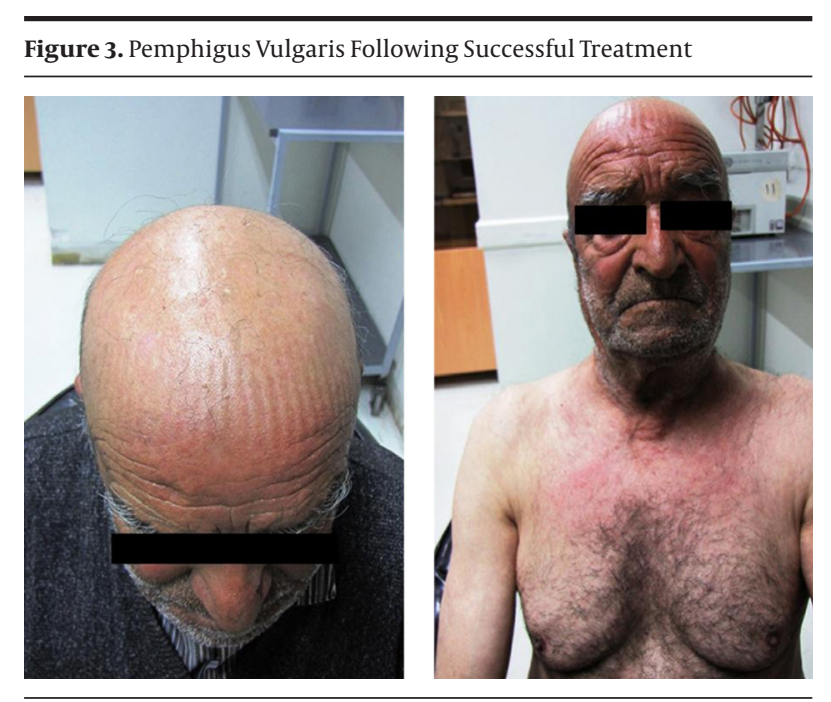

Wound healing and tissue repair of the scalp and trunk in the patient following four-year successful treatment with prednisolone and azathioprine.

ic therapy with the oral prednisolone at an initial dosage of $70 \mathrm{mg} /$ day ( $1 \mathrm{mg} / \mathrm{kg} /$ day), and azathioprine to reduce the inflammatory response and autoantibody production. He was advised to follow his treatment. Accordingly, the consent form was obtained from the patient and the study protocol conformed to the ethical guidelines of the 1975 Declaration of Helsinki as reflected in a prior approval by the Institution's Human Research Committee; the patient was followed for four years from October 2008 to October 2012. In October 2012, he was admitted to a local Clinic and the dose of prednisolone was slowly tapered down to $12.5 \mathrm{mg} /$ day (a decrease of $5 \mathrm{mg}$ every two weeks). Following physical examination, interestingly, the past lesions were completely healed (Figure 3 ) and it clearly demonstrated complete remission achieved four years from diagnosis. Accordingly, the skin biopsy was taken 


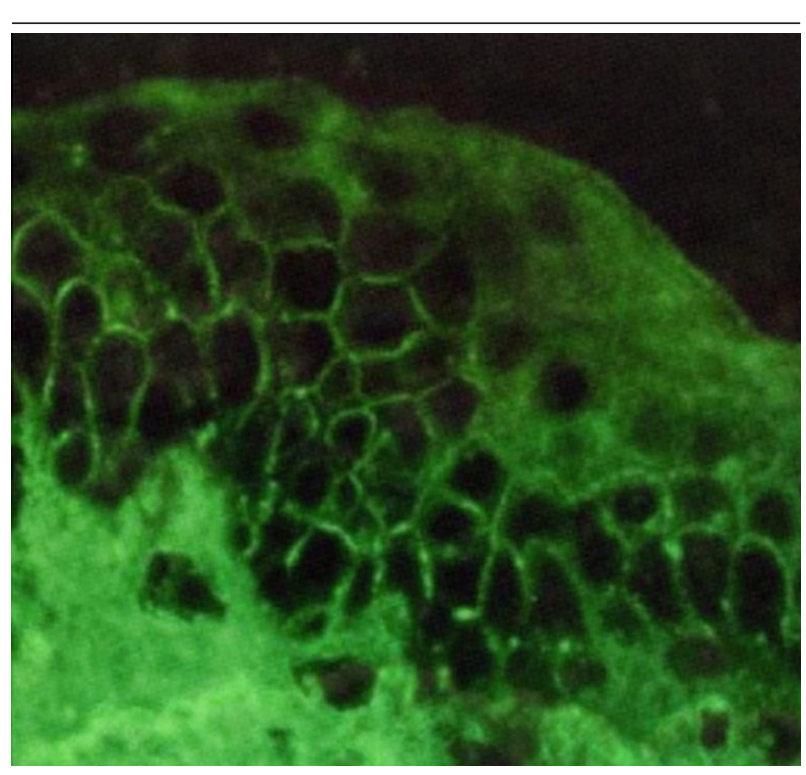

Figure 4. Direct immunofluorescence microscopy (DIF) of the cutaneous lesions revealed weak in vivo IgG deposition on the keratinocyte cell surface from the mid to upper epidermal layers (weakly positive lace like pattern in the epidermis, original magnification $\mathrm{x} 100$ ).

from the erythematous back, and the test showed the weak in vivo IgG deposition at the intercellular level in the stratum basale, stratum spinosum, and the basement membrane in the healed area of the skin (Figure 4). The patient remained under the treatment of $10 \mathrm{mg} /$ day and $100 \mathrm{mg} /$ day of prednisolone and azathioprine, respectively. He also received 1000-mg/day calcium and 400-IU/ day vitamin D3 for the treatment of osteoporosis for the following two months.

\section{Discussion}

We reported a case of PV in an elderly patient with the extensive mucocutaneous lesions and his successful treatment. We have recently shown that the mean age of patients at PV onset is 40.19 years in the central provinces of Iran; the youngest patient in our study was 17 years old. Although Langan et al. have reported that the median age at presentation in the UK is 71 years old (range, 21-102) (6), our finding were consistent with the finding of Asilian et al. who have found the mean age at onset of 41.1 years in the central areas of Iran (4). On the other hand, Martel et al. reported that PV incidence displays two peak in the third and sixth decades of life (5). In addition, several other studies have shown that PV occurs most frequently at the fifth decade of life and not in the elderly $(7,8)$. Moreover, we must stress that the elderly people are prone to development of the blistering disorders, especially the autoimmune bullous disorders such as the bullous pemphigoid. Although the main reason is not fully known, it could be partially explained by the immunologic dysregulation that occurs with aging. Another potential reason is the differences in the skin of elderly patients in comparison with the younger adults' skin. In fact, one study suggested that chemical induction of early blisters oc- curs more readily in the elderly skin in comparison with the younger adults' skin (9).

$\mathrm{PV}$ is a serious disease that needs treatment as it is almost fetal if left untreated. Treatment consists of corticosteroids, gold-containing drugs, or immunosuppressor medications such as azathioprine and methotrexate. Plasmapheresis and the monoclonal anti- $\mathrm{CD}_{20}$ antibody may be used in addition to the systemic drugs to decrease the concentration of antibodies in the bloodstream (10). Unfortunately, the use of high-dose systemic corticosteroids is associated with complications such as osteoporosis and corticosteroid-induced hyperglycemia (11).

Prognosis is not very promising in the patients with the extensive PV and in the elderly patients. Our patient was an elderly man and used a combination of prednisolone and azathioprine for four years; however, he survived and only showed osteoporosis, which might be due to longterm consumption of prednisolone; however, aging is another important risk factor for osteoporosis. The osteoporosis in this patient would be due to a combination of prednisolone therapy and aging. In fact, there were two reasons for the complete remission of this patient. The first one was taking medication. The patient received the initial low-dose oral prednisolone at the concentration of $70 \mathrm{mg} /$ day for ten days while he was hospitalized. Subsequently, the prednisolone dose was reduced to $50 \mathrm{mg} /$ day until two months prior to readmission to the hospital. The reason for this decrease was minimizing the risk of potential side effects (3). Based on the clinical examinations, and laboratory tests, the patient experienced osteoporosis from his first visit (2008) through the follow-up visit (2012). He recently developed runny eyes; however, longer follow-up may reveal other side effects. As mentioned above, two months before readmission, a $5 \mathrm{mg}$ reduction of prednisolone every two weeks was suggested by the local physician; however, a report has suggested a $50 \%$ reduction every two weeks once healing is induced and continued with healing of most lesions. In addition, Herman et al. have suggested a reduction of 5 to $10 \mathrm{mg}$ of prednisolone per week and more slowly less than $20 \mathrm{mg}$ prednisolone per day; however, the dosing schedules are vastly experimental and are adjusted on the practical experience and patients' situation (3). This means that the patient should be followed up. Ultimately, the treatment may be withdrawn if there is prolonged clinical healing.

The second probable reason was that the patient was very old and since the immune system is suppressed with age and PV is immune-mediated, we may also conclude that the disease has subsided in our case due to the agerelated immune suppression.

\section{Acknowledgements}

The authors would like to thank the staff of the Clinical Dermatology and Laboratory Diagnostic of the Isfahan Al-Zahra Hospital for assisting in the preparation of this work. We would also like to thank the Research Deputy of the Isfahan University of Medical Sciences, Iran, for the financial support of this work. Finally, we are grateful to 
Mr. Ali Saffaei from the Pharmacy Students' Research Committee, School of Pharmacy, Isfahan University of Medical sciences, for his aid in the manuscript preparation.

\section{Authors' Contributions}

Study concept and design: Mohsen Masjedi, Ali Asilian, and Zabihollah Shahmoradi. Analysis and interpretation of data: Mohsen Masjedi, Ali Asilian, Parvin Rajabi, and Bahareh Abtahi-Naeini. Drafting of the manuscript: Mohsen Masjedi, Parvin Rajabi, and Ali Asilian. Critical revision of the manuscript for important intellectual content: Bahareh Abtahi-Naeini, Mohsen Masjedi, Ali Asilian, and Parvin Rajabi.

\section{Funding/Support}

This study was supported by the grant No. 83404 from the Isfahan University of Medical Sciences.

\section{References}

1. Duvert-Lehembre S, Joly P. [Autoimmune blistering diseases]. Rev Med Interne. 2014;35(3):166-73.

2. Ruocco V, Ruocco E, Lo Schiavo A, Brunetti G, Guerrera LP, Wolf R. Pemphigus: etiology, pathogenesis, and inducing or triggering factors: facts and controversies. Clin Dermatol. 2013;31(4):374-81.

3. Harman KE, Albert S, Black MM, British Association of D. Guidelines for the management of pemphigus vulgaris. Br J Dermatol. 2003;149(5):926-37.

4. Asilian A, Yoosefi A, Faghini G. Pemphigus vulgaris in Iran: epidemiology and clinical profile. Skinmed. 2006;5(2):69-71.

5. Martel P, Cordel N, Courville P, Gilbert D, Musette P, Joly P. Pemphigus with clinical, histological and immunological features of both vulgaris and foliaceus subtypes. Br J Dermatol. 2002;147(6):1263.

6. Langan SM, Smeeth L, Hubbard R, Fleming KM, Smith CJ, West J. Bullous pemphigoid and pemphigus vulgaris-incidence and mortality in the UK: population based cohort study. BMJ. 2008;337:a180.

7. Ikeda S, Imamura S, Hashimoto I, Morioka S, Sakuma M, Ogawa $\mathrm{H}$. History of the establishment and revision of diagnostic criteria, severity index and therapeutic guidelines for pemphigus in Japan. Arch Dermatol Res. 2003;295 Suppl 1:S12-6.

8. Shamim T, Varghese VI, Shameena PM, Sudha S. Pemphigus vulgaris in oral cavity: clinical analysis of 71 cases. Med Oral Patol Oral CirBucal. 2008;13(10):E622-6.

9. Parker SR, MacKelfresh J. Autoimmune blistering diseases in the elderly. Clin Dermatol. 2011;29(1):69-79.

10. Kasperkiewicz M, Schmidt E, Zillikens D. Current therapy of the pemphigus group. Clin Dermatol. 2012;30(1):84-94.

11. Alavi A, Lowe J, Walsh S, Juurlink D, Mortaz-Hedjri S, Shear NH Corticosteroid-induced hyperglycemia is increased 10-fold in patients with pemphigus. Int J Dermatol. 2012;51(10):1248-52. 